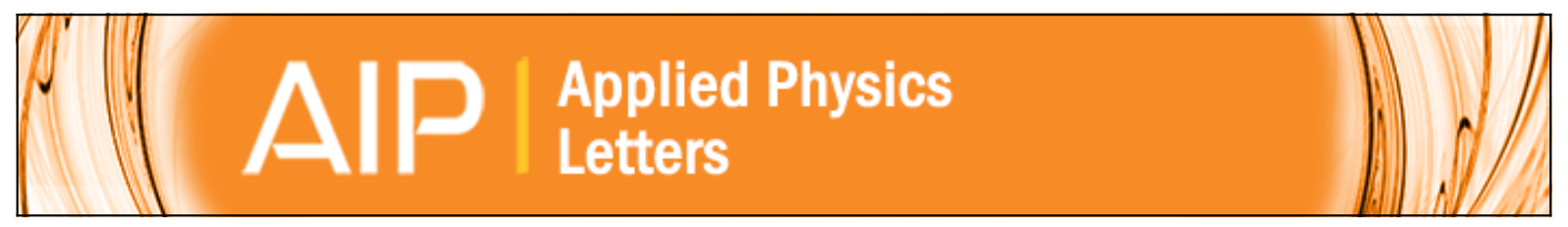

\title{
Extraordinary Hall effect and ferromagnetism in Fe-doped reduced rutile
}

Zhenjun Wang, Wendong Wang, Jinke Tang, Le Duc Tung, Leonard Spinu, and Weilie Zhou

Citation: Applied Physics Letters 83, 518 (2003); doi: 10.1063/1.1593825

View online: http://dx.doi.org/10.1063/1.1593825

View Table of Contents: http://scitation.aip.org/content/aip/journal/apl/83/3?ver=pdfcov

Published by the AIP Publishing

\section{Articles you may be interested in}

Mn-doped amorphous Si:H films with anomalous Hall effect up to $150 \mathrm{~K}$

Appl. Phys. Lett. 94, 072507 (2009); 10.1063/1.3089247

Carrier induced ferromagnetism in $\mathrm{Nb}$ doped $\mathrm{Co}$ : Ti O 2 and $\mathrm{Fe}$ : Ti O 2 epitaxial thin film

J. Appl. Phys. 99, 08M121 (2006); 10.1063/1.2177194

Co-doped anatase Ti O 2 heteroepitaxy on $\mathrm{Si}(001)$

J. Appl. Phys. 97, 073511 (2005); 10.1063/1.1868854

Growth and properties of molecular beam epitaxially grown ferromagnetic Fe-doped TiO 2 rutile films on TiO 2 (110)

Appl. Phys. Lett. 84, 3531 (2004); 10.1063/1.1703845

Ferromagnetism and transport properties of Fe-doped reduced-rutile TiO 2- $\bar{\delta}$ thin films

J. Appl. Phys. 93, 7870 (2003); 10.1063/1.1556122

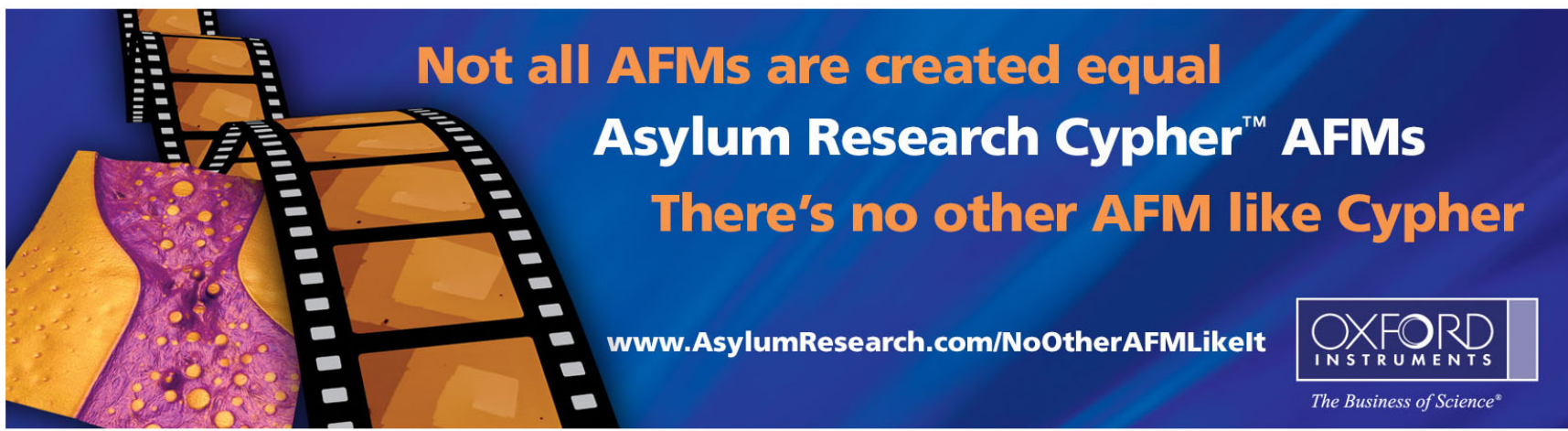




\title{
Extraordinary Hall effect and ferromagnetism in Fe-doped reduced rutile
}

\author{
Zhenjun Wang, Wendong Wang, and Jinke Tang ${ }^{\text {a) }}$ \\ Department of Physics, University of New Orleans, New Orleans, Louisiana 70148 \\ Le Duc Tung, ${ }^{\text {b) }}$ Leonard Spinu, and Weilie Zhou \\ Advanced Materials Research Institute, University of New Orleans, New Orleans, Louisiana 70148
}

(Received 31 March 2003; accepted 21 May 2003)

\begin{abstract}
Room-temperature ferromagnetism is observed in reduced rutile $\mathrm{TiO}_{2-\delta}$ by $\mathrm{Fe}$ doping. The epitaxial films grown by pulsed-laser deposition are carefully examined by x-ray diffraction, transmission electron microscopy, and magnetic and transport measurements. The films exhibit the extraordinary Hall-effect and thin-film magnetic shape anisotropy. The magnetic moments and anticipated Curie temperatures of the films rule out Fe particles, iron oxides, and Ti-Fe oxides as possible sources for the observed magnetic signals. The carriers of the Fe-doped reduced rutile are $p$-type, with a carrier density of $1 \times 10^{22} / \mathrm{cm}^{3}$. This room-temperature dilute magnetic semiconductor should find potential applications in spintronics. (C) 2003 American Institute of Physics. [DOI: 10.1063/1.1593825]
\end{abstract}

Dilute magnetic semiconductors have received considerable attention because of their combined magnetic and transport properties, which are desirable for spintronics applications. ${ }^{1-5}$ Recently, it has been reported that ferromagnetic state exists in Co-doped anatase $\mathrm{TiO}_{2}$ semiconductors at RT. ${ }^{6}$ X-ray absorption spectroscopy study on Co-doped anatase films suggests that $\mathrm{Co}$ is in the $2+$ oxidation state rather than forming metal clusters. ${ }^{7}$ RT ferromagnetism has also been indicated when rutile, another common structure of $\mathrm{TiO}_{2}$, is doped with Co. ${ }^{8,9}$ However, there has been no report on the magnetotransport properties of these systems to confirm that the carriers are indeed coupled to the magnetism. It has been controversial as to whether Co-doped $\mathrm{TiO}_{2}$ is a true magnetic semiconductor or if the effect is due to the clustering of magnetic dopant. ${ }^{10}$ Here, we report RT ferromagnetism in Fe-doped $\mathrm{TiO}_{2-\delta}$ films of reduced rutile structure by pulsed-laser deposition (PLD). Extraordinary Hall effect (EHE) observed in the films suggests that the carriers interact with the ferromagnetism. Another important result of our study is that the carriers of this magnetic semiconductor are $p$-type, contrary to the $n$-type reported for Co-doped anatase $\mathrm{TiO}_{2}$.

Stoichiometric rutile $\mathrm{TiO}_{2}$ crystallizes in a tetragonal lattice and is easily reduced by forming oxygen vacancies or titanium interstitials. Large concentrations of vacancies and/or interstitials condense into platelets that are removed from the structure by forming Magnéli shear planes. ${ }^{11,12}$ The shear planes are (101) planes relative to the stoichiometric rutile tetragonal lattice, and the reduced rutile forms a homologous series of compounds $\operatorname{Ti}_{n} \mathrm{O}_{2 n-1}(n=4, \ldots, 10)$, with triclinic structure.

$\mathrm{Fe}_{x} \mathrm{Ti}_{1-x} \mathrm{O}_{2-\delta}(x=0.02,0.06$, and 0.08$)$ thin films were epitaxially grown on (012) oriented $\alpha-\mathrm{Al}_{2} \mathrm{O}_{3}$ substrates by PLD. The films were prepared in vacuum at a substrate temperature of $980 \mathrm{~K}$. The pressure during the deposition was $1 \times 10^{-6}$ Torr. The pulsed excimer laser uses $\mathrm{KrF}(\lambda$

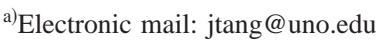

${ }^{b}$ Current address: Department of Physics, University of Warwick, Coventry CV4 7AL, UK.
}

$=248 \mathrm{~nm})$ and produces a laser beam of intensity of $1-2 \mathrm{~J} / \mathrm{cm}^{2}$ and repetition rate of $4-10 \mathrm{~Hz}$. The deposition rate is between 0.2 and $0.7 \AA / \mathrm{s}$, and the film thickness varies from 120 to $300 \mathrm{~nm}$.

$\mathrm{X}$-ray diffraction (XRD) results indicate that the films are single phase and of reduced rutile type with (202) plane, relative to the stoichiometric rutile tetragonal cell, parallel to the film plane. Figure 1(a) shows the $\theta-2 \theta$ XRD scans of the samples $\mathrm{Fe}_{x} \mathrm{Ti}_{1-x} \mathrm{O}_{2-\delta}(x=0.02,0.06$, and 0.08). The (101) and (202) reflections of the stoichiometric rutile are also shown as two vertical lines. The main peak at the center of the scans is that of the substrate. The (202) peaks are shifted toward lower $2 \theta$ angles and are present in all three samples. However, the (101) peak is absent in samples $x=0.02$ and 0.08 due to substantial reduction that forms a Magnéli shear plane. The degree to which sample $x=0.06$ is reduced is different than the other two; however, both (101) and (202) peaks are shifted toward lower angles, which is consistent with the formation of reduced rutile, ${ }^{13}$ but may also be influenced by the Fe doping.

Transmission electron microscopy (TEM) shows that the films are epitaxially grown [see Fig. 1(b)], where the lattice planes of the film and substrate are clearly seen and their orientations well defined. A selected area diffraction pattern indicates that the films have the triclinic structure of $\mathrm{Ti}_{n} \mathrm{O}_{2 n-1}$.

Vibrating sample magnetometer measurements show the films are ferromagnetic at room temperature. Figure 2 shows the magnetic hysteresis curves taken at RT. If one assumes that only Fe contributes to the magnetization (see subsequent discussion), the magnetic moment at a field of $10 \mathrm{kOe}$ is about 2.4 $\mu_{\mathrm{B}}$ per Fe atom for samples $x=0.02$ and 0.06 , and is slightly lower $\left(2.3 \mu_{\mathrm{B}}\right)$ for sample $x=0.08$. The coercivity ranges from 200 to 900 Oe. The lower-right inset of Fig. 2 compares the hysteresis of $\mathrm{Fe}_{0.06} \mathrm{Ti}_{0.94} \mathrm{O}_{2-\delta}(x=0.06)$ measured with field applied parallel and perpendicular to the film plane. The shape anisotropy associated with the film geometry is evident, which suggests that the magnetic signals originate from the film itself instead of from isolated mag- 

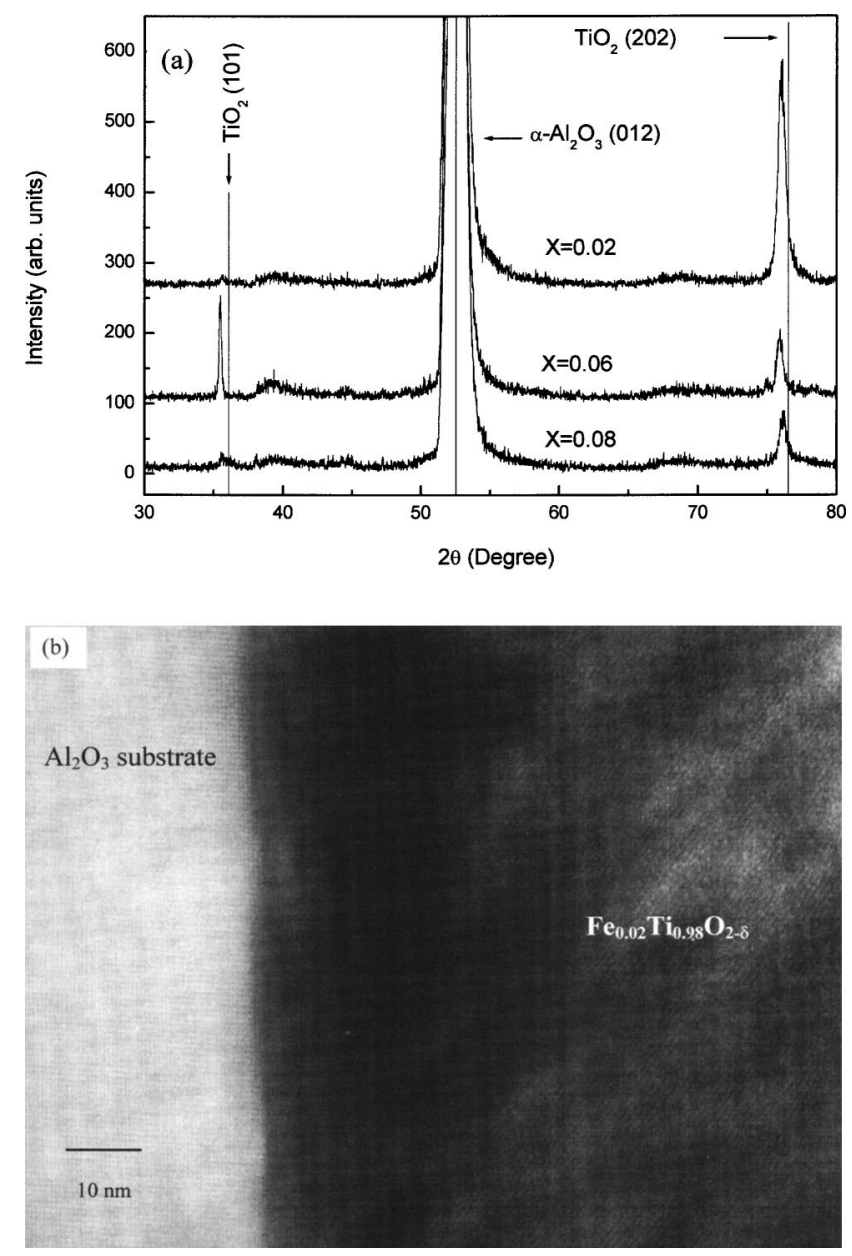

FIG. 1. (a) XRD scans of the Fe-doped reduced rutile films $\mathrm{Fe}_{x} \mathrm{Ti}_{1-x} \mathrm{O}_{2-\delta}$ $(x=0.02,0.06$, and 0.08). (b) High-resolution electron microscopy image ( $x=0.02$ ) showing epitaxial growth of the film on (012) oriented $\alpha-\mathrm{Al}_{2} \mathrm{O}_{3}$ substrate.

netic impurities. The other two samples exhibit similar anisotropy.

The upper-left inset of Fig. 2 shows the temperature dependence of the magnetization $M$ of sample $x=0.02 . M$ starts to fall as the temperature is near $400 \mathrm{~K}$, suggesting that the system is approaching the Curie temperature, which makes it unlikely that $\mathrm{Fe}$ metal particles $\left(T_{\mathrm{C}}=1045 \mathrm{~K}\right)$ be the magnetic impurity. It is impossible for iron oxides like $\mathrm{Fe}_{3} \mathrm{O}_{4}$ and $\gamma-\mathrm{Fe}_{2} \mathrm{O}_{3}$ to give rise to the magnetic signals because, in addition to the Curie temperature discrepancy, their moments are too small $\left(\leqslant 1.3 \mu_{\mathrm{B}} / \mathrm{Fe}\right)$ compared to our samples. Ti-substituted iron oxides have even smaller magnetic moment per Fe atom. The magnetization of the ferromagnetic component of parasitic ferromagnets $\alpha-\mathrm{Fe}_{2} \mathrm{O}_{3}$ and $\mathrm{FeTiO}_{3}$ is extremely small, much smaller than $\sim 1 \mu_{\mathrm{B}} / \mathrm{Fe}$. The solid solution between $\alpha-\mathrm{Fe}_{2} \mathrm{O}_{3}$ and $\mathrm{FeTiO}_{3}$ has a comparable moment over some composition range, but only at low temperatures.

The films exhibit increasing resistivity with decreasing temperature (see Fig. 3). The inset shows the details of the resistivity curves in the high-temperature region. They are almost temperature-independent near room temperature. The carrier density obtained from the Hall measurements (see subsequent discussion) is about $\sim 10^{22} / \mathrm{cm}^{3}$ (holes). The combination of the semiconducting behavior and high carrier

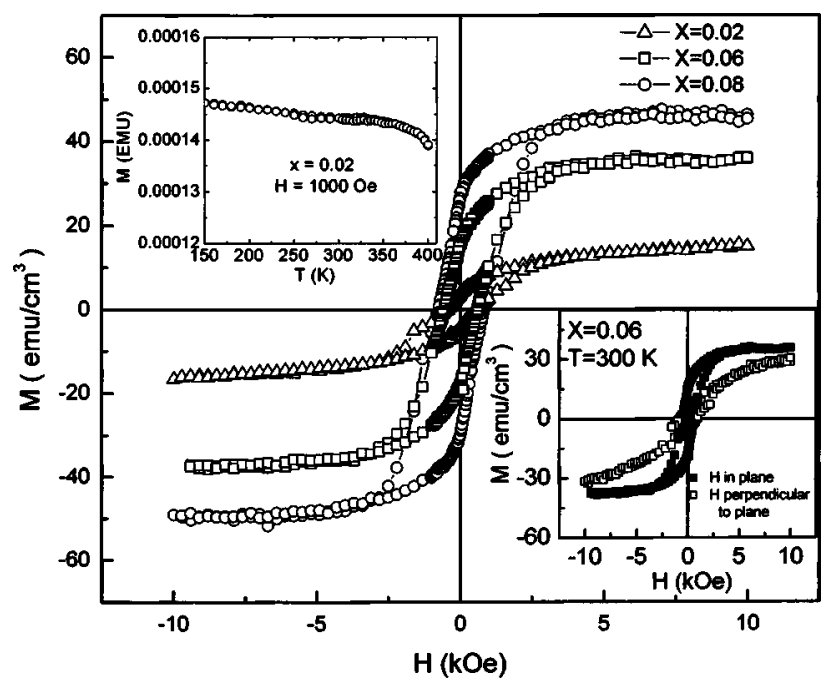

FIG. 2. The magnetic hysteresis curves of the Fe-doped reduced rutile films taken at RT. The lower-right inset compares the hysteresis loops $(x=0.06)$ measured with field applied parallel and perpendicular to the film plane. The upper-left inset shows the temperature dependence of the magnetization for sample $x=0.02$.

density suggests the films are compensated semiconductors. The compensation allows the samples to remain semiconducting at a higher carrier density compared to the uncompensated samples. ${ }^{14}$ We are currently trying to obtain reliable data on the temperature dependence of the carrier densities to further understand this behavior. Nevertheless, the temperature dependence of the resistivity of our samples is consistent with that of a compensated semiconductor: it follows exponential temperature dependence at low temperature and saturates at RT. The RT resistivity increases slightly from $\rho$ $=0.0015$ to $0.0022 \Omega \mathrm{cm}$ as $x$ changes from 0.02 to 0.08 .

The Hall effect of the films was measured with a fourprobe method at room temperature. All three films exhibit EHE associated with a ferromagnet. Figure 4 shows the Hall hysteresis loops for the three samples. The current used is different for each sample: $I=50,400$, and $500 \mu \mathrm{A}$ for samples $x=0.02,0.06$, and 0.08 , respectively. The EHE exhibits hysteresis loops with coercivities corresponding to those in the magnetic hysteresis loops. Our data suggest that

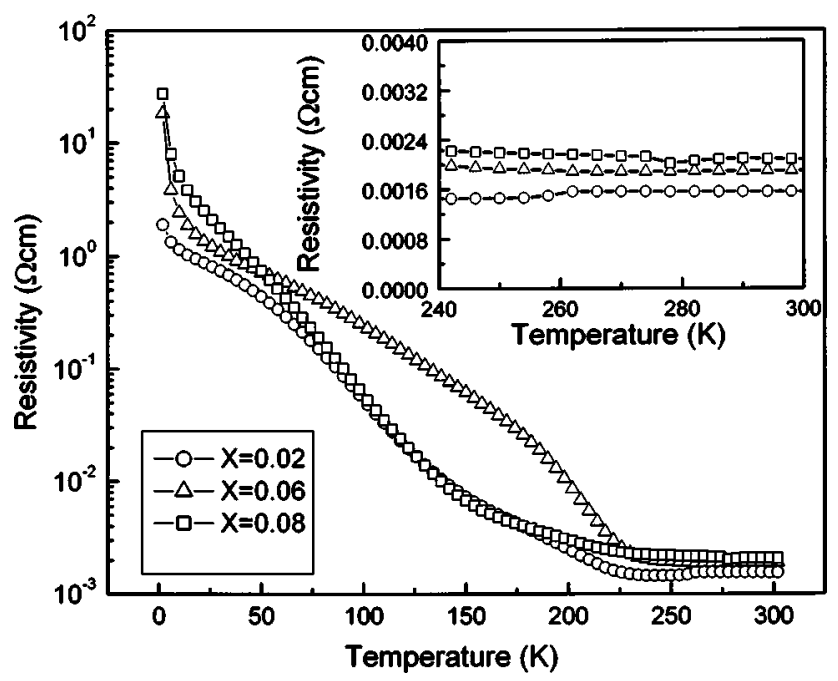

FIG. 3. The resistivity as a function of temperature. The inset shovs the details of the resistivity curves in the high-temperature region. 


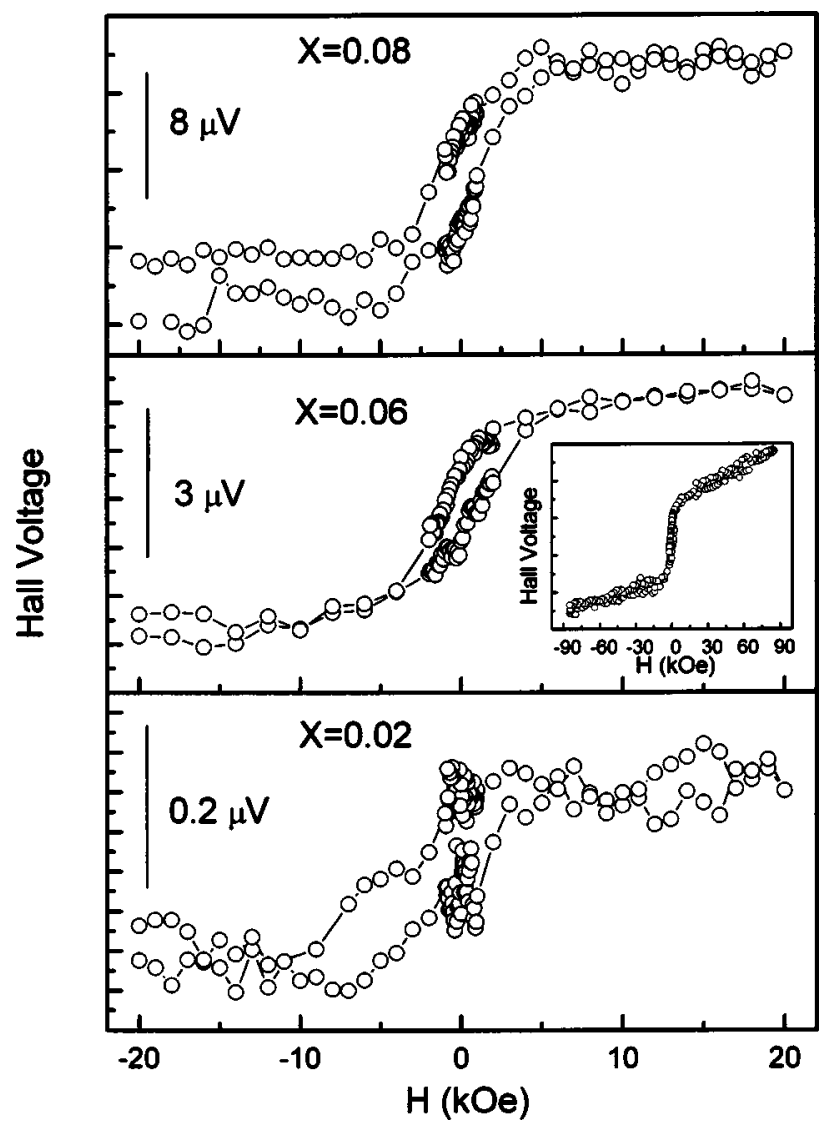

FIG. 4. EHE of the Fe-doped reduced rutile films at room temperature. The inset of the middle panel shows the Hall voltage as a function of applied magnetic field in the high-field region.

the Fe-doped reduced-rutile films are indeed ferromagnetic semiconductors. The observation of the EHE indicates that the carriers are strongly coupled to the magnetism of the films. It should be mentioned here that EHE gives one of the most conclusive pieces of evidence for a true magnetic semiconductor. ${ }^{15}$

It is not possible for the magnetic nanoparticles imbedded in a nonmagnetic medium to give rise to EHE in our films. Were they responsible for the EHE, the coercivity in the Hall hysteresis would have been zero or very small because the particles have to be sufficiently small (a few nanometers) to lead to significant scattering. This contradicts the large RT coercivity observed in our films. Careful TEM investigation has not found any nanoparticles or impurity phases in the samples.

The magnetic-field dependence of the sheet resistance of the films does not show giant magnetoresistance (GMR) typically associated with a granular system. GMR is expected and has always been observed in systems in which magnetic nanoparticles are claimed to give rise to the EHE. ${ }^{16-18}$ The absence of GMR in our films suggests it is impossible that magnetic nanoparticles/impurities are imbedded in a nonmagnetic matrix.

The carriers of the magnetic semiconductors are $p$-type, which is determined from the ordinary Hall effect observed at high fields. The inset of the middle panel of Fig. 4 shows the Hall voltage as a function of applied magnetic field in the high-field region $(-85 \mathrm{kOe}<H<85 \mathrm{kOe})$ at $300 \mathrm{~K}$ for $\mathrm{Fe}_{0.06} \mathrm{Ti}_{0.94} \mathrm{O}_{2-\delta}$. The carrier density of $1 \times 10^{22} / \mathrm{cm}^{3}$ is esti- mated from the slope of the high-field data. It ought to be noted that $n$-type carrier was reported for Co-doped anatase $\mathrm{TiO}_{2} ;{ }^{7}$ however, this material has yet to demonstrate EHE.

The origin of the magnetism in our films is not understood, but might be related to the Zener-type hole-mediated Ruderman-Kittel-Kasuya-Yashida-like interaction, which is responsible for the known magnetic semiconductor such as (Ga,Mn)As. ${ }^{19-21}$ The large hole concentration in our samples may have played an important role in the coupling between the Fe magnetic moments that gives rise to the RT ferromagnetism. When one pays attention to the unique structure of the reduced rutile, which produces $\mathrm{Ti}^{3+}$ ions with a $3 d$ moment, both $\mathrm{Fe}$ and $\mathrm{Ti}$ may contribute to the magnetization. The possible involvement of $\mathrm{Ti}(3 d)$ electrons makes this system even more interesting, and its understanding possibly more challenging. Photoemission experiments in $\mathrm{Ti}_{4} \mathrm{O}_{7}$ have shown the formation of $3 d$ band at the Fermi level at RT, which shifts to below the Fermi level at low temperature. ${ }^{22}$ The Fe-doped $\mathrm{Ti}_{n} \mathrm{O}_{2 n-1}$ may have the top of the $3 d$ band located near or below the Fermi energy. The current carriers in such situations can be expected to involve holes.

This work was supported by Sharp Laboratories of America and Louisiana Board of Regents Support Fund, Grant No. LEQSF(2000-03)-RD-B-10.

${ }^{1}$ G. A. Prinz, Science 282, 1660 (1998).

${ }^{2}$ P. Grünberg, Phys. Today 54, 31 (2001).

${ }^{3}$ G. Schmidt and L. W. Molenkamp, J. Appl. Phys. 89, 7443 (2001).

${ }^{4}$ T. Dietl, J. Appl. Phys. 89, 7437 (2001).

${ }^{5}$ H. Ohno, A. Shen, F. Matsukura, A. Oiwa, A. Endo, S. Katsumoto, and Y. Iye, Appl. Phys. Lett. 69, 363 (1996).

${ }^{6}$ Y. Matsumoto, M. Murakami, T. Shono, T. Hasegawa, T. Fukumura, M. Kawasaki, P. Ahmet, T. Chikyow, S. Koshihara, and H. Koinuma, Science 291, 854 (2001).

${ }^{7}$ S. A. Chambers, Materials Today, 5, 34 (2002); S. A. Chambers, S. Thevuthasan, R. F. C. Farrow, R. F. Marks, J. U. Thiele, L. Folks, M. G. Samant, A. J. Kellock, N. Ruzycki, D. L. Ederer, and U. Diebold, Appl. Phys. Lett. 79, 3467 (2001).

${ }^{8}$ Y. Matsumoto, R. Takahashi, M. Murakami, T. Koida, X. J. Fan, T. Hasegawa, T. Fukumura, M. Kawasaki, S. Koshihara, and H. Koinuma, Jpn. J. Appl. Phys. 40, L1204 (2001).

${ }^{9}$ W. K. Park, R. J. Ortega-Hertogs, J. S. Moodera, A. Punnoose, and M. S. Seehra, J. Appl. Phys. 91, 8093 (2002).

${ }^{10}$ D. H. Kim, J. S. Yang, K. W. Lee, S. D. Bu, T. W. Noh, S.-J. Oh, Y.-W. Kim, J.-S. Chung, H. Tanaka, H. Y. Lee, and T. Kawai, Appl. Phys. Lett. 81, 2421 (2002).

${ }^{11}$ S. Anderson, B. Collen, V. Kuylenstierna, and A. Magneli, Acta Chem. Scand. 11, 1941 (1957).

${ }^{12}$ J. B. Goodenough, in Progress in Solid State Chemistry, edited by H. Reiss (Pergamon, New York, 1971), Vol. 5, p. 145.

${ }^{13}$ S. Andersson and L. Jahnberg, Ark. Kemi 21, 413 (1964).

${ }^{14}$ B. I. Shklovskii and A. L. Efros, Sov. Phys. JETP 34, 435 (1972).

${ }^{15}$ T. Jungwirth, Q. Niu, and A. H. MacDonald, Phys. Rev. Lett. 88, 207208 (2002).

${ }^{16}$ A. Gerber, A. Milner, M. Karpovsky, B. Lemke, H.-U. Habermeier, J. Tuaillon-Combes, M. Negrier, O. Boisron, P. Melinon, and A. Perez, J. Magn. Magn. Mater. 242/245, 90 (2002).

${ }^{17}$ P. Xiong, G. Xiao, J. Q. Wang, J. Q. Xiao, J. S. Jiang, and C. L. Chien, Phys. Rev. Lett. 69, 3220 (1992).

${ }^{18}$ H. Sato, Y. Kobayashi, Y. Aoki, and H. Yamamoto, J. Phys.: Condens. Matter 7, 7053 (1995).

${ }^{19}$ T. Dietl, H. Ohno, F. Matsukura, J. Cibert, and D. Ferrand, Science 287, 1019 (2000).

${ }^{20}$ J. König, H.-H. Lin, and A. H. MacDonald, Phys. Rev. Lett. 84, 5628 (2000).

${ }^{21}$ M. Berciu and R. N. Bhatt, Phys. Rev. Lett. 87, 107203 (2001).

${ }^{22}$ M. Abbate, R. Potze G. A. Sawatzky, C. Schlenker, H. I. Lin, L. H. Tjeng, C. T. Chen, D. Teehan, and T. S. Turner, Phys. Rev. B 51, 10150 (1995). 